6. Clusters of Galaxies, Cooling Flows 


\title{
X-RAY SPECTRA OF CLUSTERS OF GALAXIES
}

\author{
Craig L. Sarazin \\ Department of Astronomy \\ University of Virginia \\ Charlottesville, Virginia U.S.A.
}

\begin{abstract}
X-ray line observations of clusters of galaxies have shown that the Xray emission in clusters is mainly thermal emission from hot diffuse gas, and that much of this gas has come out of stars, probably having been ejected from galaxies in the cluster. Future high resolution observations should allow us to determine the physical state of the gas. X-ray line measurements and abundance determinations can lead to strong constraints on the origin of the intracluster gas, and on the chemical evolution and history of galaxies. Some of the stronger resonant X-ray lines may be observable as absorption lines against a background quasar. Such X-ray absorption line measurement can be used to directly derive distances to clusters, using a technique similar to (and possibly complementary to) that the well-known method using the Zel'dovich-Syunyaev effect.
\end{abstract}

\section{INTRODUCTION}

The study of the X-ray emission from clusters of galaxies is an area of astrophysics where X-ray spectroscopy has already played an essential role. In the first case, the discovery of iron K lines in the spectrum of the Perseus cluster (Mitchell et al., 1976), followed by their discovery in the Coma and Virgo clusters (Serlemitsos et al., 1977), established immediately that the emission mechanism of clusters was thermal emission. This implied that all of the apparently empty space between the galaxies in clusters was actually filled by diffuse, hot intracluster gas with typical temperatures and electron densities of $T \sim 7 \times 10^{7} \mathrm{~K}$ and $n_{e} \sim 10^{-3} \mathrm{~cm}^{-3}$. The total mass of this gas is found to somewhat exceed the total mass of all of the galaxies in the clusters. The strength of the iron $\mathrm{K}$ lines indicated that the iron abundances in these three clusters were about half of the solar value. Since the only known sources for the production of heavy elements are in the interiors of stars, and the only significant populations of stars are located in galaxies, this indicated that much of the intracluster gas had come out of stars and may have been been ejected from galaxies. Since the present rates of stellar mass loss in galaxies are generally thought to be at least two orders of magnitude too small to have produced this gas, this suggested that galaxies may have had much more star formation and mass loss in the past.

A second case where X-ray line spectroscopy has played an essential role in clusters was the discovery of low ionization X-ray lines emitted at the centers of some clusters of galaxies (Canizares et al, 1988; Mushotzky 1984). This discovery, made with the Einstein X-ray Observatory, indicated that there are considerable amounts of cooler gas $\left(T \sim 10^{6}-10^{7} \mathrm{~K}\right)$ at the cluster centers. It is thought that this gas originates in "cooling flows" of gas moving in from the intracluster medium. While 
there is also considerable evidence supporting the cooling flow picture from X-ray images of clusters, the X-ray spectroscopy is still the most difficult piece of evidence for alternative theories. Although cooling flows provide some of the most interesting applications of X-ray spectroscopy to clusters, they are reviewed by Fabian in this volume and I will not discuss them further. I will concentrate on the applications of $\mathrm{X}$-ray spectroscopy to the hotter gas filling the bulk of the volume in clusters.

In this volume, there are several excellent reviews of the atomic processes by which X-ray lines are emitted, and I will not review this subject. The gas density is very low and the radiation field is very weak in clusters, and as a result, there are no direct spectral diagnostics for the density of the gas. The likely time scales for changes in the physical conditions in clusters are very long, and one therefore expects the gas to be in collisional ionization equilibrium. The emission lines are certainly not very optically thick, although resonant scattering may be somewhat important and can produce observable absorption lines toward background quasars (see $\$ 4$ below). Under these conditions, the X-ray spectrum of clusters depends only on the electron temperature and abundances in the gas. At the temperatures found in clusters, the strongest emission lines are expected (and observed) to be the iron $\mathrm{K}$ lines (from $\mathrm{Fe}$ XXV, Fe XXVI, and satellites from lower ions) at photon energies of about $7 \mathrm{keV}$, the iron L lines (from Fe XVII to Fe XXIV) at about $1 \mathrm{keV}$, and the $\mathrm{K}$ lines of the lighter elements $\mathrm{O}, \mathrm{Ne}, \mathrm{Mg}, \mathrm{Si}, \mathrm{S}, \mathrm{Ar}$, and $\mathrm{Ca}$ (Sarazin and Bahcall 1977).

\section{EXISTING OBSERVATIONS、}

Because the Einstein X-ray Observatory was not sensitive to $7 \mathrm{keV}$ photons, all of the observations of the Fe $\mathrm{K}$ lines have been made with proportional counters or gas scintillation proportional counters with poorer spatial resolution. The $\mathrm{Fe} \mathrm{K} \alpha$ line ( $n=2$ to $n=1$, where $n$ is the principal quantum number) has been observed in about 30 clusters. Major surveys include the OSO-8 survey of Mushotzky et al. (1978), the Ariel-5 survey of Mitchell et al. (1979), and the HEAO A-1 survey of Mushotzky (1984) and Henriksen and Mushotzky (1985). Many of the results have been compiled by Rothenflug and Arnaud (1985), and reviewed by Mushotzky $(1984,1988)$. The Fe $\mathrm{K} \alpha$ observations all indicate iron abundances in the intracluster gas of about $1 / 2$ of the solar value (Mushotzky 1984,1988; Henriksen and Mushotzky 1985; Rothenflug and Arnaud 1985). In the present data, there is no significant evidence that the iron abundance depends on any cluster property, such as richness and X-ray luminosity. Of course, the number of clusters observed is small enough that it is difficult to rule out any such variation. Henriksen and Mushotzky (1985) found a slight tendency for the iron abundance to be higher in lower temperature clusters, although this effect was not seen in the compilation of Rothenflug and Arnaud (1985), and might be due to errors in the atomic physics used to calculate the line emission.

Recently, gas scintillation proportional counters on EXOSAT and Tenma have been used to observe the spectra of clusters of galaxies (Hughes et al., 1988a,b; Singh et al., 1986; Okumura et al. 1988). These instruments have about twice the spectral resolution of normal proportional counters. This higher resolution is particularly useful for the study of the iron $\mathrm{K} \beta$ line $(n=3$ to $n=1)$. The presence of this line confirms the thermal excitation of the iron lines, and the ratio of $\mathrm{Fe} \mathrm{K} \beta / \mathrm{K} \alpha$ should be useful as a diagnostic for the temperature in the gas. The best observations of this ratio would appear to be those of Okumura et al. (1988) for the Coma, Perseus, and Ophiuchus clusters. However, these observations pose a serious problem, since the $\mathrm{K} \beta / \mathrm{K} \alpha$ ratio measured for Coma and Ophiuchus is higher than that which can be 
produced at any temperature assuming thermal excitation and collisional ionization equilibrium.

Observations of $\mathrm{K}$ lines from intermediate elements such as $\mathrm{Mg}, \mathrm{Si}$, and $\mathrm{S}$ and of the L lines of Fe have been made with the Solid State Spectrometer (SSS) on the Einstein X-ray Observatory (Lea et al., 1982; Mushotzky 1984,1988). At the SSS resolution, it is somewhat difficult to resolve these lines from the continuum, and they have been observed mainly in clusters with cooling flows where a range of gas temperatures contribute to the emission. As a result, the abundances of the intermediate elements are very uncertain, but are probably in the range of $1 / 3$ to 3 times solar (Mushotzky 1984).

The L Lines from O VIII and Fe XVII-XXIV have been observed at higher resolution with the Focal Plane Crystal Spectrometer (FPCS) on the Einstein Xray Observatory (Canizares et al., 1988). Only a few lines in the nearest clusters were strong enough to be detected. Because these lines are mainly produced by relatively cool gas, only clusters with cooling flows were detected. In these clusters, a range of gas temperatures contribute to the line emission, making the abundance determination more difficult. Nonetheless, the intensities of oxygen and iron lines in M87/Virgo and Perseus clusters strongly suggest that the ratio of oxygen to iron abundances in these clusters is about three times the solar value (Canizares et al., 1988).

One important question is whether the heavy elements in the intracluster gas are spread uniformly throughout the cluster, or are concentrated in the center of the cluster. All of the abundances quoted above assume that the composition of the intracluster gas is uniform. If the heavy elements were concentrated in the center of the cluster, their emission per unit mass would be increased because of the higher electron density, and the required abundances might be decreased by as much as a factor of $\sim 20$. There are at least two reasons why the heavy elements might concentrate in the cluster core. First, the heavy elements would eventually settle at the bottom of the cluster gravitation potential unless their diffusion were impeded or the gas were kept well mixed (Fabian and Pringle 1977; Rephaeli 1978; Abramopoulos et al., 1981). Alternatively, the heavy element enriched gas may come only from galaxies, and may be deposited primarily in the center of the cluster because the higher ram pressure there is more effective in removing the gas from galaxies (Nepveu 1981). Although the present $X$-ray line observations lack the spatial resolution to map out the heavy element abundances in any detail, there are now a number of cases where the observations are not consistent with any very strong heavy element abundance gradients (Lea et al., 1982; Ulmer et al. 1987; Hughes et al., 1988b).

In the future, it should be possible to determine the temperature of the intracluster gas from line ratio diagnostics, preferably from two or more lines from the same ion. At present, the gas temperature in clusters is usually determined from the shape of the continuum X-ray spectrum. Because the gas in clusters is hot and because the Einstein and EXOSAT telescopes were insensitive to hard X-rays, most of the temperature data come from low spatial resolution proportional counters. These observations mainly provide global or average gas temperatures. Such global temperature measurement can be used to assess the importance of heating processes in the gas in clusters. Except for the small cooling flow regions at the centers of some clusters, the cooling time in the intracluster gas is generally longer than the Hubble time. Thus, the intracluster gas can retain any heat it acquires, either during its formation or subsequently. If the intracluster gas either falls into the cluster, or is ejected without much extra energy from galaxies, it will initially have a thermal energy per unit mass which is comparable to the depth of the gravitation potential well in the cluster 
(see, for example, Sarazin 1988a). Then, one would expect the gas temperature to satisfy $T \approx \mu m_{p} \sigma^{2} / k \propto \sigma^{2}$, where $\mu$ is the mean mass per particle in the gas in units of $m_{p}$ and $\sigma$ is the cluster velocity dispersion. Mushotzky (1988) has shown that the observed relation between $T$ and $\sigma$ has a flatter slope than $T \propto \sigma^{2}$, albeit with a great deal of scatter. Although part of the scatter and flattening might be due to errors in the determination of cluster velocity dispersions, if the trend found by Mushotzky is real, it suggests that other heating processes might play a role. Such processes include heating by supernovae during the ejection of gas from galaxies, heating by nonthermal particles associated with cluster radio sources, and heating by drag on the galaxy motions. In any case, if heating occurs it is apparently most important in the poorer clusters with smaller velocity dispersions.

In addition to average temperatures, it is very important to determine the spatial variation of the gas temperatures in clusters. Since the intracluster gas is hydrostatic, one can derive the total mass and dark matter distribution in clusters from the radial variation of the gas temperature and gas density (Fabricant and Gorenstein 1983). The radial variation of the temperature also tells us to what extent thermal conduction has modified the temperature distribution in clusters. Theoretically, one expects that if conduction is not suppressed by magnetic fields, it will make the central $\sim 1 \mathrm{Mpc}$ of the cluster gas isothermal, but will not affect the temperatures of the outermost gas. Observational limits on the temperature variation in the Coma cluster by Hughes et al. (1988b) are consistent with this sort of variation.

\section{ABUNDANCES AND THE EVOLUTION OF THE INTRACLUSTER GAS}

The heavy element abundances in the intracluster gas provide many interesting constraints on the origin and evolution of this gas, and on the history of star formation and the chemical evolution of galaxies. The intracluster gas shows the integrated effect of the large number of galaxies in the clusters, and thus gives a global picture of galactic evolution which may compliment the more detailed information acquired by studying individual galaxies.

Several of the most obvious lessons from the intracluster gas abundances were mentioned in the introduction. A significant amount of the intracluster gas must have come out of stars. Now, at present the only significant population of stars we observe are located in galaxies; this suggests that a significant part of the intracluster gas came out of galaxies. Another possible source might be a generation of pregalactic stars ("Population III"), but since the intracluster gas abundances are higher than those of many galactic stars, it is unlikely that such pregalactic stars contributed much to the heavy elements in the intracluster medium. Since stellar mass loss rates from the present stellar population in the elliptical and S0 galaxies are rather low, this also suggests that these galaxies had many more massive stars and more star formation in the past.

One simple way to parameterize the effect of stellar and galactic ejection is to consider the intracluster gas to be a mixture of unprocessed, primordial gas and of processed gas ejected from galaxies. A number of calculations of the evolution of the stellar populations of elliptical galaxies suggest that they will produce gas with heavy element abundances of several times solar (Larson and Dinerstein 1975). This suggests that the intracluster medium may be composed of comparable amounts of primordial and ejected gas.

The total mass of iron in the intracluster gas in a typical rich cluster is about 
$M_{F e} \gtrsim 10^{11} M_{\odot}$, which is very considerable when compared to the total mass of stars in all of the galaxies in a typical cluster. This confirms the notion that these galaxies had an earlier generation which included many massive stars; it is possible that the remnants of these earlier stars contribute to the "dark matter" in galaxies and clusters. The comparison of the abundances of different heavy elements in intracluster gas can also provide useful information on the stars which produced the heavy elements. At present, the only useful information on this question comes from the observation that the oxygen to iron ratio is several times solar in the M87/Virgo and Perseus clusters (Canizares et al., 1988). It is believed that oxygen is produced primarily in Type II supernovae from short-lived massive stars, while much of the iron production may occur in Type I supernovae involving older, lower mass stars. The high $\mathrm{O} / \mathrm{Fe}$ ratio in clusters provides another indication that high mass stars were more common in the past and contributed significantly to the enrichment of the intracluster gas.

From the observed mass of iron in the intracluster gas one can estimate the nuclear energy released in the fusion leading to the iron; this gives $E_{n u c l} \gtrsim 10^{63} \mathrm{ergs}$, which is comparable to the gravitational binding energy of all of the intracluster gas. For massive stars, part of this energy appears as optical radiation, and part appears as kinetic energy in stellar winds and supernovae. Unless the overwhelming majority of this energy is radiated away, the heating due to supernovae and winds from the massive stars which produced the heavy elements in clusters will be very significant. This heating will be most important in smaller systems (galaxies and poorer groups), which have shallower gravitational potential wells. There should be enough energy to easily remove most of the gas from galaxies, and to remove part of the gas from poorer groups. Thus, one would expect to find a higher ratio of gas to stellar mass in rich clusters than in poor clusters or in individual elliptical galaxies. In fact, Rothenflug and Arnaud (1985) and Mushotzky (1988) find that the ratio of $M_{\text {gas }}$ to $M_{\text {stars }}$ does increase with $M_{\text {star }}$ for clusters. Moreover, the remaining gas in galaxies or poor clusters should have been significantly heated. One would expect to find that the gas in poorer groups and individual elliptical galaxies is hotter relative to the velocity dispersion than in richer clusters. In $\S 2$, I noted that this appears to be the case for poorer clusters, and the ratio of $T / \sigma^{2}$ is even higher for ellipticals. As a result of this, one would expect the gas in elliptical galaxies and in poorer clusters to have a flatter density distribution (slower decrease with radius) than in rich cluster.

The picture presented above represents one very simple scenario for the chemical evolution of galaxies and clusters. In this volume, Forman, Jones, and David present a somewhat different model in which the variation in the ratios of $M_{\text {gas }}$ to $M_{\text {stars }}$ represents a variation in the efficiency of galaxy formation with environment, with rich clusters being less efficient at galaxy formation. Future high resolution X-ray line spectroscopy can distinguish between these two models. For example, the FormanJones-David models predicts that the heavy element abundances should be greater in poor clusters and in elliptical galaxies. In general, one would like to measure the abundances of several different elements in a wide range of clusters and in individual elliptical galaxies. For at least a sample of nearby clusters, it is important to map out the spatial variation of the line emission to determine whether the heavy elements are uniformly distributed throughout the gas. Finally, it is very important to extend these spectral observations to clusters at significant redshifts. Such observations should constrain the time at which the heavy elements were produced. 


\section{X-RAY ABSORPTION LINES AND THE DISTANCE TO CLUSTERS}

Several authors have noted that the strongest resonance X-ray lines from clusters may be slightly optically thick (Shapiro and Bahcall 1980; Basko et al., 1981; Gil'fanov et al., 1987). Basko et al. suggested that these resonance lines might be detectable in absorption in the X-ray spectrum of a background quasar behind a cluster of galaxies. Detailed calculations show that some ions should have column densities of of at least $10^{16} \mathrm{~cm}^{2}$, leading absorption line equivalent widths of several $\mathrm{eV}$ (Basko et al. 1981; Sarazin 1988b). The strongest lines are generally the Fe XXVI $1 s-2 p$ and Fe XXV $1 s^{2} S-1 s 2 p^{1} P$ lines. Gil'fanov et al. suggested that the lines would be somewhat optically thick. The optical depth of the lines is more difficult to predict than the absorption equivalent width, since the optical depth depends inversely on the line width. The minimum line width is that due only to thermal broadening. Because these X-ray lines are produced by heavy elements, the thermal line widths are quite narrow (a small fraction of the sound speed). However, it is likely that the turbulent line broadening exceeds thermal line broadening. The expected optical depths of the strongest lines are in the range $0.01-10$, with the larger values applying if the lines are only thermally broadened. Gil'fanov et al. argue for the larger values of the line optical depth, and suggest that resonant scattering might affect the line profiles and the surface brightness distributions of the lines.

If an X-ray absorption line due to intracluster gas could be detected in the spectrum of a background quasar and if the same line could be observed in emission from the cluster, this would allow a direct determination of the distance to the cluster. The reason this leads to a distance measurement is simple; absorption is independent of distance, as long as the absorber lies between the observer and the background light source. The observed flux due to emission decreases with distance squared, and so comparing absorption and emission leads to a distance estimate. The arguments leading to the distance estimator for cluster X-ray absorption lines are very similar to those which have been applied to the Zel'dovich-Syunyaev effect in clusters (Zel'dovich and Syunyaev 1969; Cavaliere et al., 1977,1979; Gunn 1978; Silk and White 1978). I learned at this conference that the same idea of using X-ray absorption lines to determine distances has been developed independently by Krolik and Raymond (see the article by Krolik in this volume).

The basic idea can been described easily; a more rigorous treatment is given in Sarazin (1988b). Let us assume that an X-ray-bright quasar is found which is located behind an X-ray emitting cluster of galaxies. We assume that the gas in the cluster is homogeneous (not highly clumped) and spherically symmetric. If the gas is not spherically symmetric, it would be necessary to observe a sample of clusters and average the results. Using a spectroscopic detector with a small aperture, the X-ray spectrum of the quasar is observed and is found to contain an absorption feature from a resonance line from an ion located in the hot gas of the cluster. Let $W$ be the equivalent width of the line in the quasar spectrum. Let us assume that the optical depth of the line is not large. Then, the equivalent width determines the column density of the ion $N_{\text {ion }}$ as

$$
\left(\frac{N_{\text {ion }}}{10^{16} \mathrm{~cm}^{-2}}\right)=0.911 f^{-1}(1+z)\left(\frac{W}{\mathrm{eV}}\right)
$$

where $z$ is the redshift of the cluster and $f$ is the absorption oscillator strength of the 
line. The column density is given by

$$
N_{\text {ion }} \equiv \int n_{\text {ion }} d l
$$

where $n_{i o n}$ is the number density of the ion and $l$ is the path length through the cluster along the line-of-sight to the quasar.

Let us consider the emission by the cluster gas in the same resonance line as the absorption. The emissivity of the cluster gas in such a line can be written as

$$
\epsilon_{\text {line }}=n_{\text {ion }} n_{e} \Lambda_{\text {line }}(T),
$$

(see, for example, Sarazin and Bahcall 1977), where $n_{e}$ is the electron density and $\Lambda_{\text {line }}$ is a function of the electron temperature $T$ which is determined by atomic physics. The surface brightness of the cluster in this line at the same position where the absorption line is measured is given by

$$
I_{\text {line }}=(1+z)^{-4} \int n_{\text {ion }} n_{e} \Lambda_{\text {line }}(T) d l .
$$

Then, comparing equations (2) and (3), we see that the average electron density along that line-of-sight is roughly

$$
\left\langle n_{e}\right\rangle \approx \frac{I_{\text {line }}(1+z)^{4}}{N_{\text {ion }}\left\langle\Lambda_{\text {line }}\right\rangle},
$$

where $\left\langle\Lambda_{\text {line }}\right\rangle$ is an average value along the line-of-sight.

The total X-ray emissivity (in any band) in the gas can be written as

$$
\epsilon_{X}=n_{e}^{2} \Lambda_{X}(T) ;
$$

again, $\Lambda_{X}$ is a function of the electron temperature $T$ which is determined by atomic physics. Then, the total flux of X-ray emission in this band as observed at the Earth is

$$
F_{X}=\frac{1}{4 \pi d_{A}^{2}(1+z)^{4}} \int n_{e}^{2} \Lambda_{X}(T) d V
$$

where $d_{A}$ is the angular-diameter distance to the cluster (Weinberg 1972), and $V$ is the volume of the cluster. Let us assume that the X-ray emission extends out to a characteristic radius $r_{X}$, and that the volume associated with the emission is roughly $4 \pi r_{X}^{3} / 3$. The quantity we might actually observe is the angular size of the X-ray emitting region $\theta_{X} \equiv r_{X} / d_{A}$. The flux can be written as

$$
F_{X} \approx \frac{1}{3}\left\langle n_{e}\right\rangle^{2}\left\langle\Lambda_{X}\right\rangle \theta_{X}^{3} d_{A}(1+z)^{-4} .
$$

Then, the distance to the cluster is given approximately by

$$
d_{A} \approx \frac{3 N_{\text {ion }}^{2}\left\langle\Lambda_{\text {line }}\right\rangle^{2} F_{X}}{(1+z)^{4} I_{\text {line }}^{2}\left\langle\Lambda_{X}\right\rangle \theta_{X}^{3}} .
$$


This is expression is approximate because the correct definitions of $\left\langle n_{e}\right\rangle,\left\langle\Lambda_{\text {line }}\right\rangle$, $\left\langle\Lambda_{X}\right\rangle$, and $\theta_{X}$ involve conflicting averages over the gas distribution in a cluster. Exact expressions in which the correct averages are determined directly from the X-ray surface brightness profile of the cluster are given in Sarazin (1988b). However, equation (9) is useful in that it shows fairly directly the dependence of the distance estimate on the observed quantities. For example, one notes (sadly) that distance estimate depends on the square of the quantities which are most difficult to measure $\left(N_{\text {ion }}\right.$, and $\left.I_{\text {line }}\right)$, and only linearly on the easiest quantity to measure $\left(F_{X}\right)$. A similar misfortune befalls distance estimates based on the Zel'dovich-Syunyaev effect (Silk and White 1978).

It will obviously be difficult to measure the required quantities (particularly $W$ and $I_{\text {line }}$ ) with sufficient accuracy to improve upon the present determinations of the Hubble constant $H_{0}$ or to determine the deceleration parameter $q_{0}$. However, this method involves many of the same measurements as the distance determinations based on the Zel'dovich-Syunyaev effect, and there is a chance that the X-ray absorption line measurements can be done more reliably than the microwave diminution measurements required for the other method. Although I have considered the application of this method to a cluster with a background quasar, some clusters (for example, M87/Virgo and Perseus) have AGNs with significant nonthermal X-ray luminosities at their centers. These clusters often have cooling flows, in which the lower gas temperatures can greatly increase the equivalent widths of the X-ray absorption lines. The measurements are likely to be much easier in these cases, although the interpretation of the measurements is likely to be more difficult.

\section{CONCLUSIONS}

The observations of X-ray emission lines from clusters of galaxies can provide a wealth of information concerning the physical state of the intracluster gas. From the abundances in the intracluster gas we should be able to determine the origin of the intracluster gas and to place strong constraints on the chemical evolution and star formation history of galaxies. Because clusters are spatially extended, these observations require only moderate spatial resolution (a fraction of a minute of arc). Depending on the particular observation, moderate-to-high spectral resolution is needed.

It should also be possible to detect X-ray absorption lines due to the intracluster gas in the spectra of background quasars. By comparing the equivalent width of such an absorption line and the flux from the same line seen in emission, one can determine directly the distance to the cluster. If this could be done with sufficient accuracy for a sample of clusters, such X-ray absorption line distance measurement could be used to determine $H_{o}$ and possibly $q_{o}$. Although the required X-ray observations are difficult, they are very similar to the observations which must be made to implement the Zel'dovich-Syunyaev distance estimator, and may complement that effort.

Finally, I should note that nearly everything that I have said applies as well to the hot gas in individual elliptical galaxies. In fact, since this gas is apparently cooler than that in clusters, the X-ray emission lines (and possibly, the absorption lines as well) should be even stronger than in clusters. 


\section{ACKNOWLEDGMENTS}

I would like to thank Jack Hughes, Julian Krolik, and Richard Mushotzky for useful comments, and Paul Gorenstein and Martin Zombeck for the effort they made to organize this very useful conference. This work was supported in part by NASA Astrophysical Theory Program Grant NAGW-764.

\section{REFERENCES}

Abramopoulos, F., Chanan, G., and Ku, W. 1981, Ap. J., 248, 429.

Basko, M. M., Komberg, B. V., and Moskalenko, E. I. 1981, Sov. Astr., 25, 402.

Canizares, C. R., Markert, T. H., and Donahue, M. E. 1988, in Cooling Flows in Clusters and Galaxies, ed. A. C. Fabian (Kluwer: Dordrecht), p. 63.

Cavaliere, A., Danese, L., and deZotti, G. 1977, Ap. J., 217, 6.

Cavaliere, A., Danese, L., and deZotti, G. 1979, Astr. Ap., 75, 322.

Fabian, A. C., and Pringle, J. E. 1977, M.N.R.A.S., 181, 5p.

Fabricant, D., and Gorenstein, P. 1983, Ap. J., 267, 535.

Gil'fanov, M. R., Syunyaev, R. A., and Churazov, E. M. 1987, Sov. Astr. Lett., 13, 3.

Gunn, J. E. 1978, in Observational Cosmology, ed. by A. Maeder, L. Martinet, and G. Tammann (Geneva Obs.: Geneva).

Henriksen, M. J., and Mushotzky, R. F. 1985, Ap. J., 292, 441.

Hughes, J. P., Yamashita, K., Okumura, Y., Tsunemi, H., and Matsuoka, M. 1988a, Ap. J., 327, 615.

Hughes, J. P., Gorenstein, P., and Fabricant, D. 1988b, Ap. J., 329, 82.

Larson, R. B., and Dinerstein, H. L. 1975, P.A.S.P., 87, 911.

Lea, S. M., Mushotzky, R., and Holt, S. 1982, Ap. J., 262, 24.

Mitchell, R. J., Culhane, J. L., Davison, P. J., and Ives, J. C. 1976, M.N.R.A.S., 175, $29 \mathrm{p}$.

Mitchell, R. J., Dickens, R. J., Bell-Burnell, S. J., and Culhane, J. L. 1979, M.N.R.A.S., $189,329$.

Mushotzky, R. F. 1984, Phys. Scripta, T7, 157.

Mushotzky, R. F. 1988, in Proc. NATO Summer School on Hot Astrophysical Plasmas, ed. by R. Pallavicini (Kluwer: Dordrecht), in press.

Mushotzky, R. F., Serlemitsos, P. J., Smith, B. W., Boldt, E. A., and Holt, S. S. 1978, Ap. J., 225, 21.

Nepveu, M. 1981, Astr. Ap., 101, 362.

Okumura, Y., Tsunemi, H., Yamashita, K., Matsuoka, M., Koyama, K., Hayakawa, S., Masai, K., and Hughes, J. 1988, preprint.

Rephaeli, Y. 1978, Ap. J., 225, 335.

Rothenflug, R., and Arnaud, M. 1985, Astr. Ap., 144, 431.

Sarazin, C. L. 1988a, X-ray Emission from Clusters of Galaxies, (Cambridge Univ.: Cambridge).

Sarazin, C. L. $1988 \mathrm{~b}$, preprint.

Sarazin, C. L., and Bahcall, J. N. 1977, Ap. J. Suppl., 34, 451.

Shapiro, P. R., and Bahcall, J. N. 1980, Ap. J., 241, 1.

Serlemitsos, P. J., Smith, B. W., Boldt, E. A., Holt, S. S., and Swank, J. H. 1977, Ap. J. (Lett.), 211, L63.

Silk, J., and White, S. D. M. 1978, Ap. J. (Lett.), 226, L103. 
Singh, K. P., Westergaard, N. J., and Schnopper, H. W. 1986, Ap. J. (Lett.), 308, L51.

Ulmer, M. P., Cruddace, R. G., Fenimore, E. E., Fritz, G. G., and Snyder, W. A. 1987, Ap. J., 319, 118.

Weinberg, S. 1972, Gravitation and Cosmology: Principles and Applications of the General Theory of Relativity, (New York: Wiley), pp. 407-468.

Zel'dovich, Y. B., and Syunyaev, R. A. 1969, Ap. Sp. Sci., 4, 301. 